\title{
Lane-Changing Behavior Prediction Based on Game Theory and Deep Learning
}

\author{
Shuo Jia $\left(\mathbb{D},{ }^{1}\right.$ Fei Hui $\mathbb{D},{ }^{1}$ Cheng Wei $\mathbb{D}^{1},{ }^{1}$ Xiangmo Zhao, ${ }^{1}$ and Jianbei Liu ${ }^{2}$ \\ ${ }^{1}$ School of Information Engineering, Chang'an University, Xi'an 710000, China \\ ${ }^{2}$ Research and Development Center on Emergency Support Technologies for Transport, \\ CCCC First Highway Consultants Co., Ltd., Xi'an 710000, China \\ Correspondence should be addressed to Fei Hui; feihui@chd.edu.cn
}

Received 21 December 2020; Revised 26 June 2021; Accepted 23 July 2021; Published 3 August 2021

Academic Editor: Haneen Farah

Copyright (c) 2021 Shuo Jia et al. This is an open access article distributed under the Creative Commons Attribution License, which permits unrestricted use, distribution, and reproduction in any medium, provided the original work is properly cited.

\begin{abstract}
Lane changing is an important scenario in traffic environments, and accurate prediction of lane-changing behavior is essential to ensure traffic and driver safety. To achieve this goal, a vehicle lane-changing prediction model based on game theory and deep learning is developed. In the game theory component, the interaction between vehicles during lane changing is analyzed according to the running state of the vehicle, with the probability of lane changing as its output. For the deep-learning component, long short-term memory and a convolutional neural network are used to extract and learn data features during the lane-changing process as well as combine the output of the game theory component to obtain the prediction result of whether the vehicle will change lanes. By using an open-source traffic dataset to train and verify the proposed model, the verification results show that the prediction accuracy can reach $94.56 \%$ within $0.4 \mathrm{~s}$ of lane-changing operation and that the model can achieve timely and accurate prediction of the lane-changing behavior of vehicles.
\end{abstract}

\section{Introduction}

Drivers' driving operations are closely related to road traffic safety; approximately $92.9 \%$ of the total traffic accidents are caused by improper driving operations [1]. Among all driver operations, operations in the lateral direction of the vehicle (left and right lane changing and turning) have the greatest impact on the stability of traffic flow and cause the most accidents. Although blind-spot-warning systems and lane departure warning systems integrated into advanced driver assistance systems can reduce the probability of accidents being caused by lateral operations to a certain extent, in actual applications, these systems need to rely on the driver to use the turn signal lights correctly. However, in the United States, the utilization rate of turn signals is only approximately $44 \%$ [2], whereas in China, it is $40 \%$ [3]. Therefore, for most drivers, these systems play a limited role and cannot solve the problem of frequent accidents being caused by lateral operation. This phenomenon motivates research on drivers' driving behavior recognition and prediction.
Scholars at the University of Michigan Transportation Research Institute studied drivers' driving behavior [4]. They collected the headway data of 36 different driving objects and used neural networks for training and recognition. However, due to the limitations of technology and data, this study has limited accuracy in identifying and predicting drivers' driving behavior. In recent years, the rapid development of communication and sensor technologies has made high-quality vehicle data increasingly available. Moreover, connected vehicle technology integrates advanced vehicle-to-vehicle communications, vehicle-to-road communications, processing power, and sensing capabilities, thereby creating an environment where vehicles can collaborate with each other. This allows researchers to conduct more in-depth and accurate studies on driving behavior, such as those on the recognition of drunk driving, recognition of fatigue driving, and classification of drivers' driving styles.

Although recent studies have begun to identify and predict driving behavior, the dataset used in some of these studies comprises historical data, and consequently, they 
cannot promptly notify drivers of possible dangers. In addition, some studies have adopted shallow learning algorithms and cannot accurately predict complex lanechanging behavior. Therefore, we need to consider a method that can predict the lateral operation the driver will perform in an accurate and timely manner, using real-time accurate networking data.

In this study, we first propose a predictive model of the driver's lane-changing intention. This model uses game theory and combines the information (lane number, speed, acceleration, distance, etc.) of the target vehicle and its neighboring vehicles in its adjacent lanes to form a Stackelberg game model. This method can obtain the dynamic interaction between different vehicles in the driving process from the data and solve the problem of dynamic changes in data and environmental uncertainty in the traditional lanechanging model. By solving the game theory model, we can predict whether the surrounding environment of the target vehicle is in a state suitable for lane changing and quantify the possibility that the driver will perform a lane-changing operation. Subsequently, a deep-learning algorithm composed of long short-term memory (LSTM) and a convolutional neural network $(\mathrm{CNN})$ is established, and historical data are used to establish the training dataset of driver lateral operation. Furthermore, the deep-learning model is repeatedly trained using the training set, so as to achieve the optimal model parameters. Finally, the entire model continuously monitors the driving-state data of all the vehicles in the target road section and analyzes the lateral and longitudinal motion characteristics of the vehicle. When the driving state of any vehicle begins to meet the lanechanging characteristics, the whole model can accurately predict the vehicle's behavior in a short period of time, and the prediction result can be used to notify surrounding vehicles in time to avoid accidents to the greatest extent.

The remainder of this paper is organized as follows. Related work is reviewed in Section 2. In Section 3, we analyze the characteristics of lane-changing behavior to prepare for subsequent prediction. Section 4 first introduces the overall architecture of the algorithm and then describes the game theory used to predict the intention and the LSTMCNN model used to predict the process. The validation data, experiments, and their results are presented in Section 5. Conclusions and future work are discussed in Section 6.

\section{Literature Review}

In the research field of drivers' driving behavior, many scholars have proposed a variety of mathematical models regarding lane-changing behavior. Based on these models, they have studied the trajectory and behavior characteristics of vehicles during the lane-changing process. Zheng [5] reviewed the development of lane-changing behavior models and divided these models into two categories: models regarding the process of lane-changing decisionmaking and models that quantify the effect of lane changing on surrounding vehicles. In this study, we focus on the possibility of the driver's lane-changing operation and the change in the state of the vehicle during the lane-changing process; hence, we mainly study the first model type. Among these models, the most classic one is the vehicle lanechanging model in the urban road environment proposed by Gipps [6]. This model involves six factors of real environments: speed, presence of other vehicles, presence of the lane line, distance of the safety gap, location of the obstacle, and the driver's intention. The driver uses these conditions to consider the possibility, necessity, and expectation of lanechanging operations. Based on this model, Yang and Koutsopoulos [7] also proposed a model suitable for highways and applied it to the microscopic traffic simulator. Based on the lane departure warning system, Li-Sheng et al. [8] proposed a new safety lane-changing model in highway scenarios. In addition, Butakov and Ioannou [9], Do et al. [10], and Keyvan-Ekbatani et al. [11] proposed different lane-changing behavior models based on various scenarios.

Moreover, many algorithm models have been increasingly applied in the field of traffic. Common models include the hidden Markov model (HMM), game theory model, and machine learning algorithm model, which significantly help in solving the problems of identification, prediction, and decision evaluation. Kamrani et al. [12] used high-frequency and diverse driving data to study the generation of driving decisions and analyzed drivers' willingness to maintain constant speed, acceleration, and deceleration. Jin et al. [13] proposed a lane-changing behavior decision-making model based on the Gauss mixture hidden Markov model (GMHMM), and through data verification, the similarity between this model and the driver's real behavior was found to reach 95.4\%. In addition, many other studies have used the HMM to solve traffic problems, such as accident detection [14], prediction of braking behavior [15], trajectory map matching [16], and prediction of traffic conditions [17]. The game theory model is often used to simulate the process of decision-making, including lane-changing decisions [18-21], and application scenarios of autonomous driving technology [22]. In recent years, machine learning has been developed rapidly, and new model structures have been proposed based on the original algorithms, which have achieved better results in the field of identification and prediction. Yu et al. [23] proposed a D3 model that can identify six forms of abnormal driving behavior by using a support vector machine and neural network (NN). Zhao et al. [24] proposed a model for predicting traffic flow transformation using an LSTM network.

Furthermore, some scholars have studied the prediction of drivers' lane-changing behavior. In the study by Cheng et al. [25], an NN was adopted to capture the complexity of lane changing, and large-scale trajectory data were employed for model estimation and validation. The final prediction accuracy of the proposed model was $94.58 \%$ for the left lanechanging samples and $73.33 \%$ for the right lane-changing samples. Moreover, a back-propagation neural network model for predicting lane-changing behavior was developed by Peng et al. [26] from the results of a naturalistic on-road experiment. The proposed model could accurately predict the lane-changing behavior of drivers at least $1.5 \mathrm{~s}$ in advance, and the accuracy reached approximately 93\%. Li et al. [27] used a combination of the HMM and Bayesian filtering 
technology to identify the driver's intention to change lanes and achieved a recognition rate of $90.3 \%$ and $93.5 \%$ for left and right lane changes, respectively, thus yielding a better effect than when only the HMM is used.

Conclusions drawn from these related studies can be summarized as follows. Although there are some studies on lane-changing behavior, they separate the driver's intention and operation of lane changing. In the actual driving process, it is precisely the driver's intention to change lanes that causes subsequent lane-changing operations. Moreover, the algorithm used in the existing research is a shallow learning algorithm, which has low applicability to complex operations such as lane changing, and the prediction effect is not optimal. For high-speed vehicles, potential hazards can be predicted $0.1 \mathrm{~s}$ in advance, and accidents can be significantly reduced. Therefore, this study first analyzes the impact of the vehicle's surrounding environment on the driver's lanechanging intention. After quantifying the possibility of lane changing using a game model, the deep-learning algorithm is used to analyze the driving data and predict the driver's lane-changing behavior in a short time.

\section{Characteristics of Lane-Changing Behavior}

Compared with other common behaviors, such as acceleration and deceleration, the lane-changing behavior of vehicles is a more complex one. In general, lane-changing behavior is divided into active and passive lane changes. Active lane change is performed by the driver in expectation of a better driving environment, whereas passive lane change is performed because the vehicle needs to leave the current road, which generally occurs in a ramp area. This study only examines the active lane-changing behavior.

A complete lane-changing process can be divided into three stages. The first is the intention generation stage. The driver observes the surrounding environment of the vehicle and generates lane-changing intentions based on this information and his/her own driving habits; he/she decides whether to change lanes and the direction of lane changing (left or right) and selects the appropriate lane-changing gap. As shown in Figure 1, the subject vehicle (SV) is the target vehicle used in our research. It is worth mentioning that the $\mathrm{SV}$ is not a specific vehicle; it can be any vehicle in the specified road section. It is affected by vehicle $V 3$ in front of it in the current lane and can only be driven at a low speed. At the same time, the average speed in the adjacent lane is better than that in the current lane, with a better driving environment, and the gap between vehicles in the target lane can meet the space requirements of the SV. Consequently, the driver may choose to change lanes to obtain a better driving experience. In this process, the factors influencing the intention to change lanes include the speed and location of vehicles SV and $V 3$ in the current lane and vehicles $V 1$ and $V 2$ in the adjacent lane. Therefore, at this stage, we can use the collected vehicle driving-state data to construct a hypothetical world of traffic and use models to calculate the potential for the vehicle to change lanes.

The second is the execution stage of the lane-changing operation. The driver operates the accelerator and steering wheel to drive the vehicle from the current lane into the target lane. As shown in Figure 1, the changes in this stage are mainly reflected in the driving state of the SV, including the transformation of the speed, acceleration, and position in the longitudinal and latitudinal directions. In this study, we predict whether the driver of the SV performs a lanechanging operation by analyzing the instantaneous change in the data during the initial process of lane changing.

The last stage is the state maintenance stage. The driver adjusts the position and speed of the vehicle through subtle operations after the vehicle enters the target lane, so that the vehicle can maintain a stable state in the target lane. The SV in this process will have little impact on other vehicles, and hence, our study does not involve this part.

\section{Methodology}

4.1. Model Architecture. The complete algorithm architecture and data flow of the model are illustrated in Figure 2. After preprocessing the original data and extracting the lanechanging behavior, the game theory algorithm is used to analyze the driver's lane-changing intention, and the lanechanging intention is added to the data samples in the form of time series data. In the prediction process, LSTM and a $\mathrm{CNN}$ are used to extract the characteristics of lane-changing behavior, and the network is trained with a large amount of sample data. Finally, the prediction of uncalibrated sequence data can be achieved.

\subsection{Prediction of Lane-Changing Intention Based on Game} Theory Model. The main purpose of this stage is to analyze the driver's lane-changing intention formation process, use the data to simulate the real lane-changing environment, and select a suitable algorithm model to calculate the probability of the driver's lane-changing decision. As described in the characteristics of the lane-changing behavior, in the actual driving process, the state of the surrounding vehicles will affect the driver's lane-changing intention, and the driver's lane-changing operation will also affect the normal driving of the surrounding vehicles; that is, the lane-changing vehicle maintains an interdependent and complex interactive relationship with the surrounding vehicles. Among the many microlane-changing models, game theory in operational research can highlight the interaction between drivers and has unique advantages in analyzing the generation process of driver lane-changing intentions. Therefore, based on the game theory model, this study examines the generation of drivers' lane-changing intentions.

Game theory was originally developed as a mathematical model in the field of operations research and has been applied to other disciplines to solve problems of collaboration and competition between different objectives. In addition, some researchers have solved the autonomous driving decision planning problem by using the concept of game theory between multiple vehicles [28]. There are three main factors in the game: players, the strategies they use, and the payoff they receive from the corresponding strategies [29]. In general, the principle of game theory can be 


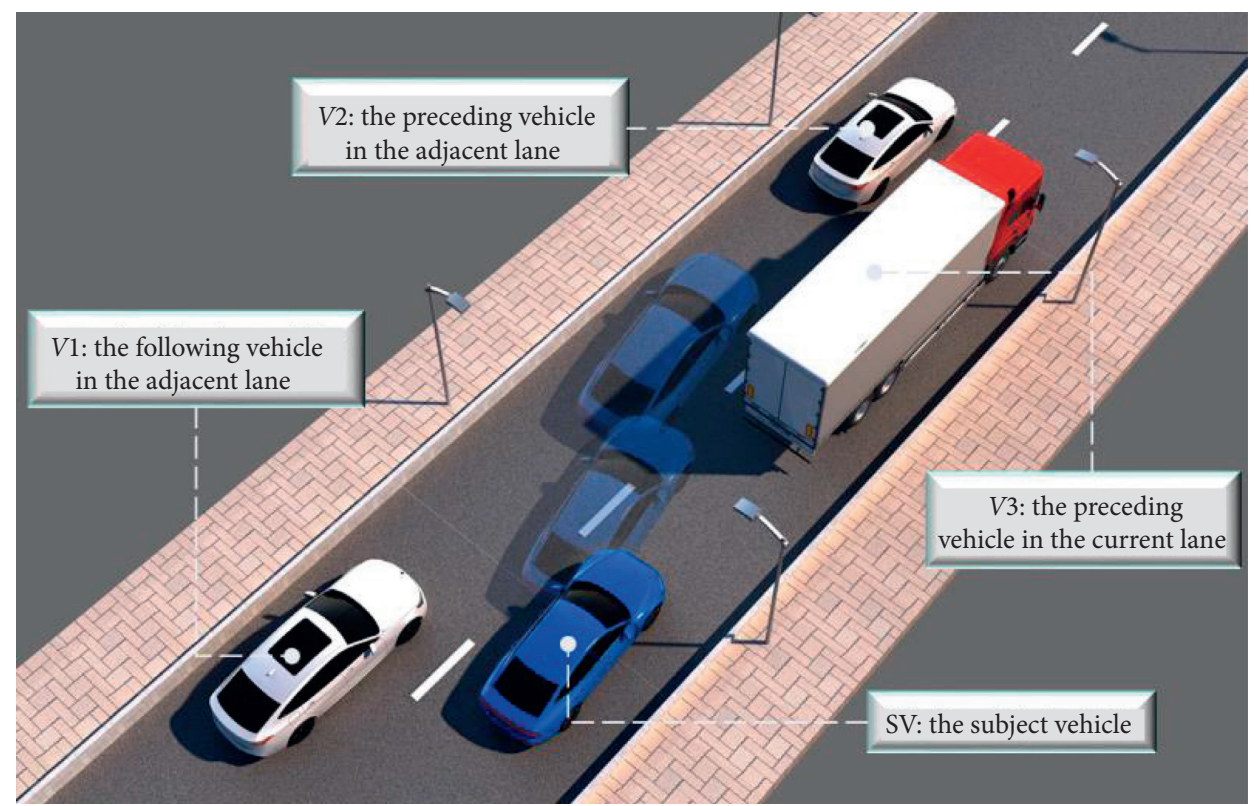

FIGURE 1: Lane-changing vehicles and surrounding environment.

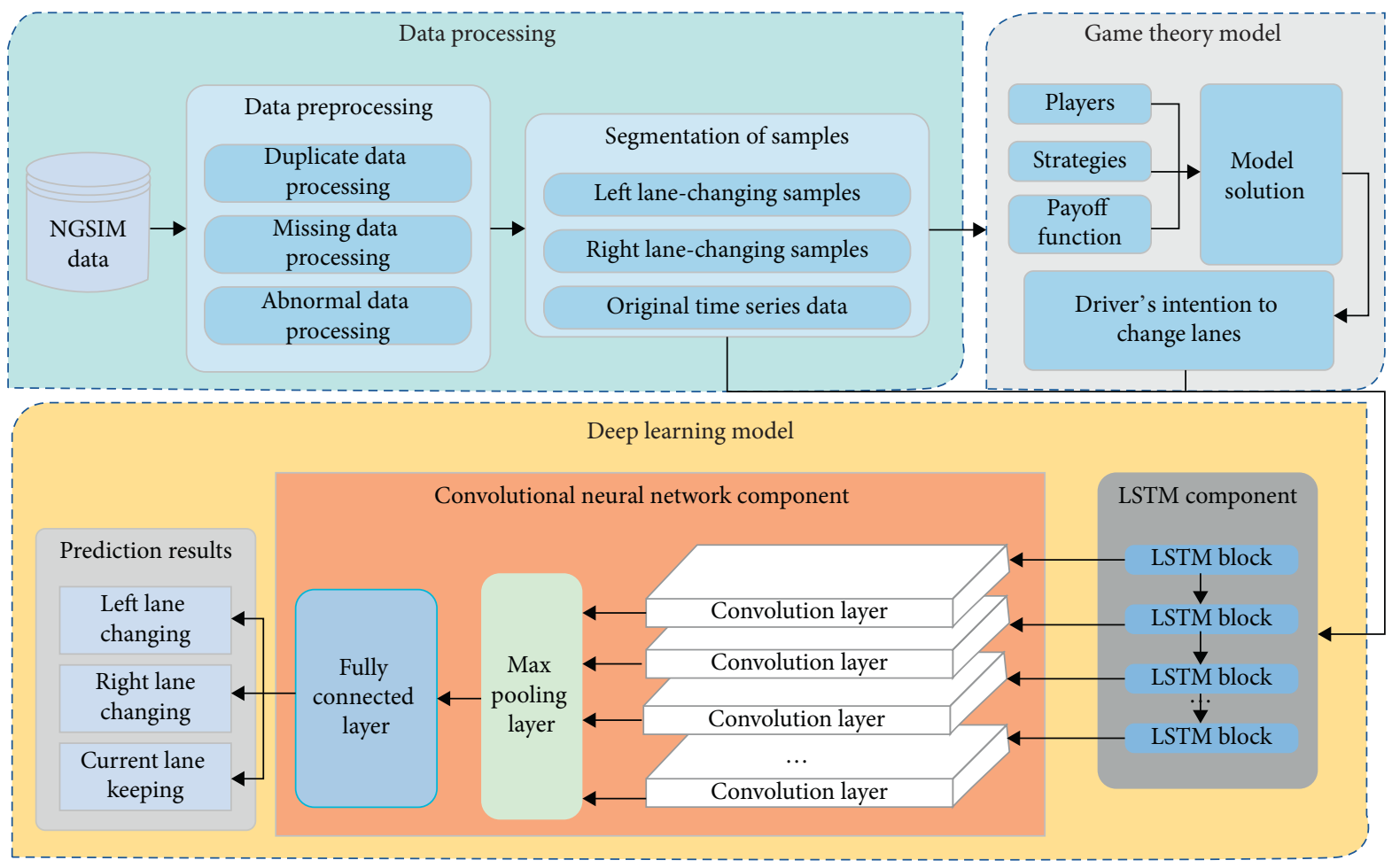

Figure 2: Model architecture and data flow.

summarized as the process by which the decision-maker makes a choice to maximize the benefit of each player after the opponent adopts a certain strategy, assuming that all players are rational. In the process of the game in this study, the drivers participating in the game do not have strong restraint rules; moreover, the drivers can observe the running status of other vehicles and make their own decisions based on this information.
As described in the previous section, the driver's decision to change lanes needs to take into account the motion state of his/her own SV and also needs to monitor the leading vehicle $V 3$ in the same lane, the leading vehicle $V 2$ in the adjacent lane, and the following vehicle $V 1$ in the adjacent lane. Among the abovementioned potentially conflicting vehicles, $V 1$ is the key factor affecting the SV. Therefore, it can be considered that these two vehicles are in a game 
relationship. For the SV, there are two strategies, namely, changing lanes and staying in the current lane. When vehicle $V 1$ encounters the SV's lane-changing decision, its strategies include keeping its current state, accelerating, or decelerating; at the same time, if the SV chooses not to change lanes, $V 1$ can also keep its current state, accelerate, or decelerate. Therefore, their strategic space can be expressed by equation (1), and the different payoffs brought by different decisions are summarized in Table 1, where $\Phi$ denotes the respective strategic space, $\tau$ stands for different decisions, and $U$ and $u$ denote the payoff brought to the SV and $V 1$ by their choice of different strategies. For example, $U_{11}$ represents the payoff brought to the SV when it chooses to change lanes, and $u_{11}$ represents the payoff brought to $V 1$ when it chooses to keep its state. The drivers of both cars want to make rational decisions to maximize their payoffs; hence, the payoff function plays an important role in game theory models. In this research, their payoff functions are mainly calculated based on two aspects of safety and space, denoted by $U_{\text {safety }}$ and $U_{\text {sapce }}$, respectively.

SV: $\Phi_{1}=\{C$ : change lanes, $S:$ stay $\}$,

$V 1: \Phi_{2}=\{K$ : keep state, $D$ : deceleration, $A$ : acceleration $\}$.

$U_{\text {safety }}$ is defined as the payoff a vehicle will obtain for keeping itself safe during the entire lane-changing process and is given by

$$
U_{\text {safety }}= \begin{cases}\frac{2|T(t)|-T_{\min }(t)}{T_{\min }(t)}, & -T_{\min }(t) \leq T(t) \leq T_{\min }(t), \\ 1, & \text { else, }\end{cases}
$$

where $T(t)$ is the time headway at time $t$ between the currently considered vehicle and the competing vehicle during lane changing and $T_{\min }(t)$ is the minimum safe time headway to ensure that the two vehicles drive in a safe state. The value of $T_{\min }(t)$ is determined by

$$
T_{\min }(t)=\min \left(T_{\text {initial }}, T_{a}\right),
$$

where $T_{\text {initial }}$ is the headway at time $t$ between $V 1$ and the preceding car $V 2$ and $T_{a}$ is set to 3 , according to a study by Qiao et al. [30], which states that the headway between vehicles should be maintained for at least $3 \mathrm{~s}$ to effectively avoid the occurrence of accidents.

$U_{\text {space }}$ represents the payoff of the vehicles in terms of the spatial distance during the lane-changing process. It is closely related to the position, speed, and acceleration of the vehicle. $U_{\text {space }}$ can be defined as

$$
U_{\text {space }}= \begin{cases}\frac{2|D(t)|-D_{\min }(t)}{D_{\min }(t)}, & -D_{\min }(t) \leq D(t) \leq D_{\min }(t), \\ 1, & \text { else, }\end{cases}
$$

TABLE 1: Lane-changing game payoff matrix.

\begin{tabular}{cccc}
\hline \multicolumn{2}{l}{ Players in the game } & \multicolumn{2}{c}{ SV } \\
& & $C$ & $S$ \\
\hline \multirow{3}{*}{$V 1$} & $K$ & $U_{11}, u_{11}$ & $U_{12}, u_{12}$ \\
& $D$ & $U_{21}, u_{21}$ & $U_{22}, u_{22}$ \\
& $A$ & $U_{31}, u_{31}$ & $U_{32}, u_{32}$ \\
\hline
\end{tabular}

where $D(t)$ is the spatial distance of the conflicting vehicles, calculated from their longitudinal and latitudinal coordinates. $D_{\min }(t)$ represents the minimum safe distance that the vehicle should maintain under normal driving conditions, which is calculated based on the speed and acceleration. $D_{\text {min }}(t)$ is given by

$$
D_{\text {min }}(t)=\max \left(\int_{0}^{t} \int_{0}^{\lambda}\left(a_{V 1}(\tau)-a_{\mathrm{SV}}(\tau)\right) \mathrm{d} \tau \mathrm{d} \lambda+\left(v_{V 1}(0)-v_{\mathrm{SV}}(0)\right) t\right),
$$

where $a_{V 1}$ and $V_{V 1}$ are the longitudinal acceleration and longitudinal velocity of $V 1$ and $a_{S V}$ and $V_{S V}$ are those of the SV, respectively.

Finally, the total payoff of the game vehicle is determined by $U_{\text {safety }}$ and $U_{\text {sapce }}$ together, and a linear function is used to express the relationship between them:

$$
U_{\text {total }}=\alpha U_{\text {safety }}+\beta U_{\text {space }},
$$

where $U_{\text {total }}$ is the total payoff of the vehicle and $\alpha$ and $\beta$ are the weight coefficients of the safety payoff and space payoff, respectively, with their sum being equal to 1 . For each driver, the weight coefficients are different, and their selection is closely related to the driver's driving style. Cautious drivers pay more attention to their own safety payoff, in which case the value of $\alpha$ is greater than $\beta$, whereas aggressive drivers pay more attention to the value of space payoff, where $\beta$ is greater than $\alpha$. In the calculation of the weight coefficient, we compare the space headway maintained by the driver on the entire road section with the average space headway of other vehicles within a range of $100 \mathrm{~m}$ and use a function similar to the sigmoid function to process the space headway difference to obtain the space weight coefficient for each driver. It can be calculated using

$$
\beta=\frac{1}{1+e^{-\left(\left|l-l_{\text {ave }}\right| / 20\right)}},
$$

where $l$ and $l_{\text {ave }}$ represent the average headway of the target vehicle and other nearby vehicles on the road section, respectively. Then, the value of $\alpha$ can be calculated from the value of $\beta$. In Figure 3, $\alpha$ and $\beta$ are shown as functions of the difference in average headway.

After the total payoff is calculated using a combination of the safety payoff and space payoff, it is introduced into the game model to find the optimal strategy. In the process of lane changing, first, the SV provides decision variables according to the driving environment; then, $V 1$ responds optimally according to the variables provided by the SV and feeds the decision back to the SV. This information circulates continuously until both sides of the game are satisfied. 


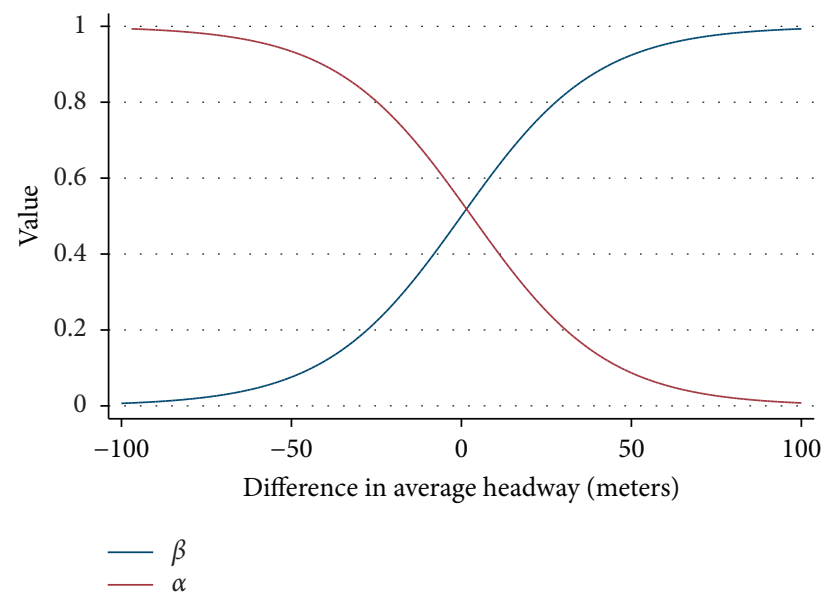

Figure 3: Weight coefficients of safety payoff and space payoff.

Therefore, this game model can be regarded as a Stackelberg game at perpetual equilibrium. This is an optimization problem that can be represented by

$$
\left\{\begin{array}{l}
\tau_{1}^{*}=\arg \max \left(U\left(\tau_{1}, \tau_{2}\right)\right), \tau_{2} \in \Phi_{2}^{\prime}\left(\tau_{1}\right), \tau_{1} \in \Phi_{1} \\
\tau_{2}^{*}=\arg \max \left(u\left(\tau_{1}^{*}, \tau_{2}\right)\right), \tau_{2} \in \Phi_{2} \\
\Phi_{2}^{\prime}\left(\tau_{1}\right) \triangleq\left\{\tau_{2}^{\prime} \in \Phi_{2}: u\left(\tau_{1}, \tau_{2}^{\prime}\right) \geq u\left(\tau_{1}, \tau_{2}\right), \forall \tau_{2} \in \Phi_{2}, \tau_{1} \in \Phi_{1}\right\}
\end{array}\right.
$$

subject to

$$
\begin{aligned}
& v_{\mathrm{SV}}, v_{V 1} \geq 0 \\
& a_{\min } \leq a_{\mathrm{SV}}, a_{V 1} \leq a_{\max },
\end{aligned}
$$

where $\tau_{1} \in \Phi_{1}$ is the decision of the SV, $\tau_{2} \in \Phi_{2}$ is the decision of $V 1, \Phi_{2}^{\prime}\left(\tau_{1}\right)$ is the set of decisions the $V 1$ can make based on the SV's decision, $\tau_{1}$ and $\tau_{2}^{*}$ are the final and best decisions the SV and $V 1$ can make based on the game model, and $a_{\min }$ and $a_{\max }$ are the minimum and maximum accelerations, set to $-4.5 \mathrm{~m} / \mathrm{s}^{2}$ and $3 \mathrm{~m} / \mathrm{s}^{2}$, respectively. Since the elements in the strategy set are finite, this optimization problem can always find the Stackelberg equilibrium by extensively searching the discrete payoff matrix (as Table 1). After obtaining the optimal strategy for the SV, the probability of changing lanes for the vehicle can be calculated using the payoff value of the current decision and the payoff value of all decisions.

4.3. Prediction of Lane-Changing Process by LSTM-CNN Algorithm. The driving-state data of vehicles belong to a type of time series data, which have continuity and correlation in time. The lane-changing behavior of a vehicle is mainly reflected in the driving-state data as the continuous change in lateral velocity, acceleration, steering angle, and coordinates. Therefore, it is necessary to select an algorithm suitable for the recognition and prediction of a time series when predicting the lane-changing process. In this paper, we propose an LSTM-CNN deep-learning algorithm model to predict the lane-changing process.
The LSTM model can selectively memorize the data entered earlier, save the data that have a significant impact on the results in a special manner, and discard the data that have little effect on the results. Because of this feature, the LSTM network has good processing capabilities for long sequences. At the same time, not all data dimensions and data fragments in the vehicle status data are useful for determining whether a vehicle is performing a lanechanging operation, and the LSTM can also filter useful information. Moreover, the $\mathrm{CNN}$ model has strong feature extraction capability for multidimensional data, which can be used to further extract data features in the lane-changing process. Therefore, in the LSTM-CNN algorithm structure proposed in this paper for predicting lane-changing behavior, the LSTM is used to first extract the key data segments related to the lane changing, after which a CNN extracts the key features of the segments. The combination of these two components can bring out the advantages of the respective algorithm structures, which can handle lanechanging processes of different time lengths while avoiding the high time consumption of the multilayer LSTM structure, which has obvious advantages in theory compared to other algorithms.

The structure of the LSTM-CNN algorithm is shown in Figure 4. The LSTM is used to complete the preliminary feature extraction related to lane changing. The $\mathrm{CNN}$ takes 


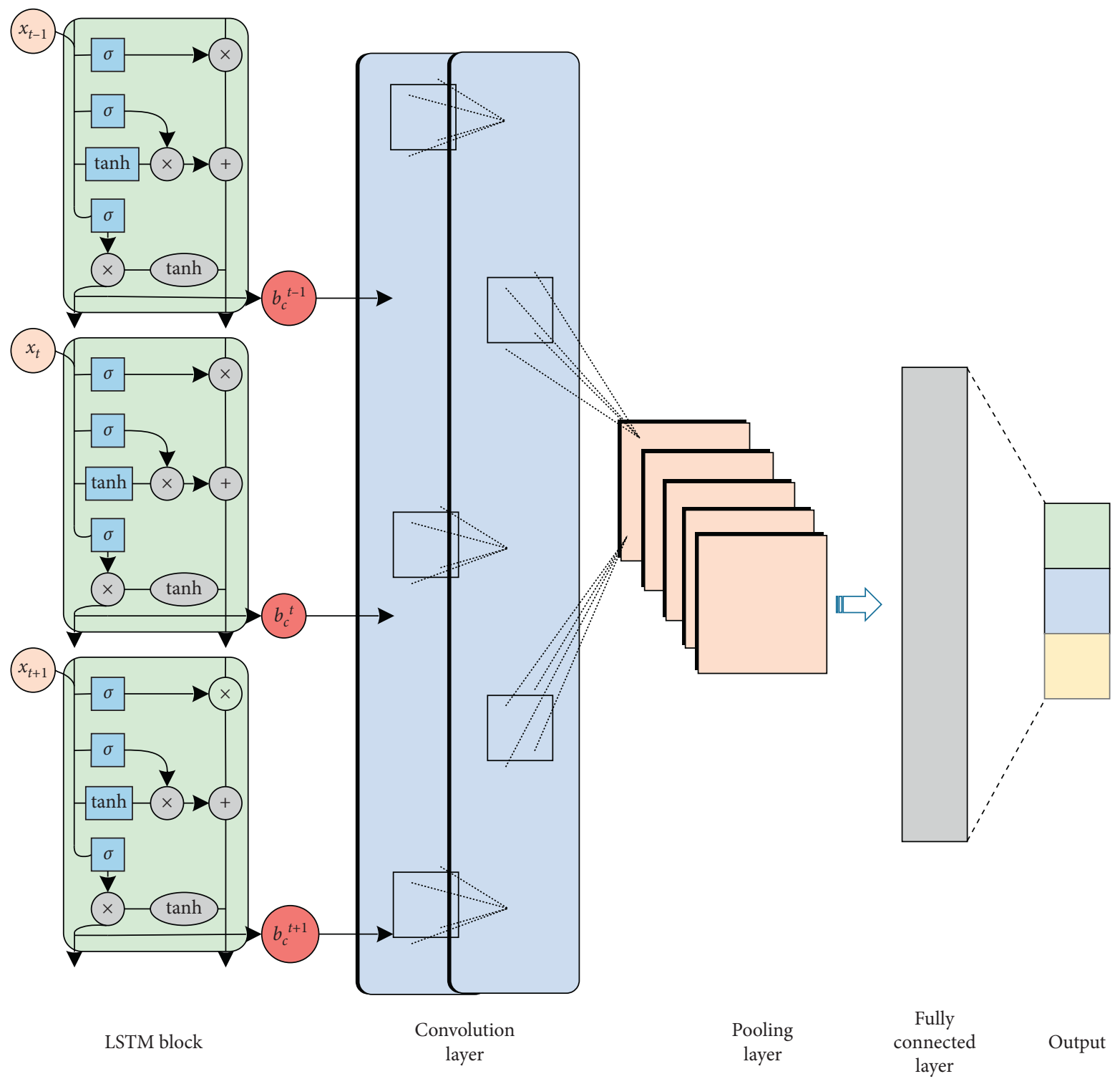

FIgURE 4: Long short-term memory and convolutional neural network model.

the output of the LSTM as its input, performs a convolution operation, further processes the extracted features, and finally outputs the prediction of whether the vehicle will change lanes.

Similar to other NNs, the LSTM algorithm model is also divided into an input layer, a hidden layer, and an output layer. The difference is that its special hidden structure can solve the vanishing gradient problem of the RNN. The hidden layer of each moment in the LSTM structure contains one or more memory blocks, each containing a cell and three gates. Among them, the cell state represents the flow of information in the block. The forget gate decides what information is discarded from the cell state at the last moment. The function of the input gate is to selectively remember new information in the cell state, and the output gate determines the output data of the cell state. The mathematical model of each structure can be expressed by the following functions:

$$
\begin{aligned}
a_{l}^{t} & =\sum_{i=1}^{I} \omega_{i l} x_{i}^{t}+\sum_{c=1}^{C} \omega_{c l} s_{c}^{t-1}, \\
b_{l}^{t} & =f\left(a_{l}^{t}\right), \\
a_{\phi}^{t} & =\sum_{i=1}^{I} \omega_{i \phi} x_{i}^{t}+\sum_{c=1}^{C} \omega_{c \phi} s_{c}^{t-1}, \\
b_{\phi}^{t} & =f\left(a_{\phi}^{t}\right), \\
a_{w}^{t} & =\sum_{i=1}^{I} \omega_{i w} x_{i}^{t}+\sum_{c=1}^{C} \omega_{c w} s_{c}^{t}, \\
b_{w}^{t} & =f\left(a_{w}^{t}\right), \\
a_{c}^{t} & =\sum_{i=1}^{I} \omega_{i c} x_{i}^{t}, \\
s_{c}^{t} & =b_{\phi}^{t} s_{c}^{t-1}+b_{l}^{t} g\left(a_{c}^{t}\right), \\
b_{c}^{t} & =b_{\omega}^{t} h\left(s_{c}^{t}\right) .
\end{aligned}
$$


In the equations above, $x_{i}^{t}$ represents the input data of each moment, which are composed of the vehicle driving state and the results of the game theory model. $s_{c}^{t-1}$ stands for the memory of information from the previous moment. $a_{l}^{t}, a_{\varphi}^{t}, a_{w}^{t}$, and $a_{c}^{t}$ are made up of input data and the cell state, and each of them represents the computation of every part of the network, including the input gate, forget gate, output gate, and memory cells. $b_{l}^{t}, b_{\varphi}^{t}, b_{w}^{t}$, and $s_{c}^{t}$ are the output of each part after different activation functions. $\omega_{i l}, \omega_{c l}, \omega_{i \varphi}, \omega_{c \varphi}, \omega_{i w}$, and $\omega_{c w}$ are the weight matrices of the network. In the learning process of the network, their values gradually tend to the best weight. The result of this component is $b_{c}^{t}$, which stands for the output of blocks.

Finally, the data are imported into the CNN component to further extract features from the LSTM results. The essence of a CNN is a multilayer perceptron, which uses local connections and shared weights to make the network easy to optimize and reduce the risk of overfitting. The most important parts of the CNN model used in this study are the convolution and pooling layers. The function of the convolution layer is to extract the characteristics of the output of LSTM component by combining with the convolution kernel. The pooling layer is used for feature selection and information filtering. The mathematical model of the $\mathrm{CNN}$ can be expressed by the following functions:

$$
\begin{aligned}
c_{i} & =f\left(W \cdot b_{c}^{t}+b\right), \\
c & =\left[c_{1}, c_{2}, \ldots, c_{n-h+1}\right], \\
\widehat{c} & =\left[\widehat{c}_{1}, \widehat{c}_{2}, \ldots, \widehat{c}_{m}\right],
\end{aligned}
$$

where $c_{i}$ is the convolution result and $W \in \mathbb{R}$ and $b \in \mathbb{R}$ are parameters in the convolution process, representing the convolution kernel and bias, respectively. $c \in \mathbb{R}$ and $\widehat{c}=\max (c)$ are simple expressions of the pooling process. After performing convolution and pooling operations, a fully connected layer is used to output the prediction results of the entire model on whether the vehicle will change lanes.

\section{Data Preparation and Experiments}

5.1. Datasets. The datasets used in this study are required to ensure high quality of continuous vehicle state time series data, simultaneously considering the interaction between vehicles. Hence, the data should include vehicle labels within a certain range. Additionally, the dataset should contain as many vehicle states as possible, including coordinates, speed, and acceleration. After comprehensive consideration of various factors, we selected the datasets of the Next Generation Simulation (NGSIM) program as the data source for this study. The datasets were provided by the Federal Highway Administration of the U.S. Department of Transportation, and the US-101 data in the datasets were used in this study. The US-101 data were collected on a segment of U.S. Highway 101 in Los Angeles, California. The data collection time was from $7: 50$ to $8: 20$ in the morning. The video of traffic flow on the road section was acquired through a camera, and the status data of vehicles were extracted using video analysis software. By preprocessing the data for this study, the unused data were cleaned up and some missing data were analyzed and supplemented. Finally, 4,098,933 data points were selected, including 2,706 cars. As summarized in Table 2, which presents the statistical data results, there are data indicating a space headway and time headway of 0 . This situation can be interpreted as the distance between vehicles being too close to be calculated from the video. It can also be regarded as valid data.

5.2. Lane-Changing Behavior in Dataset. After the dataset was preprocessed and segmented according to the vehicle ID, rules were defined to intercept the lane-changing behavior segments of the vehicle from the data. First, we calculated the coordinate distribution of all vehicles in each lane to determine the center line of each lane. Then, according to the trajectory of the vehicle from near the center line of one lane to another lane, it was determined that the vehicle changed lanes. Finally, this lane-changing behavior segment of the vehicle was sliced to obtain a sample. After slicing the lane-changing behavior of all vehicles, a total of 1,659 samples were extracted from 1,263 vehicles to form the training and verification sample sets of the research model. Among all lane-changing segments, there were 509 right lane-changing samples and 1,150 left lane-changing samples. The duration of the segments is shown in Figure 5, indicating that most vehicles completed the movement from the center line of one lane to another within 3-7s. The vehicle speed and acceleration during the lane-changing process are shown in Figure 6. In addition, 3,120 segments of lane-keeping behavior were extracted from the dataset in order to expand the training set and better recognize lanekeeping behavior; the length of these sample segments varies from 2 to $10 \mathrm{~s}$.

5.3. Building and Verification of the Proposed Model. To verify the prediction effect of the proposed model, we used a workstation to build the model. The operating system of the workstation is Windows 10, equipped with Intel Xeon CPU e5-2623V3@3 GHz, 32.0GB RAM, and NVIDIA Quadro RTX4000 GPU. The hardware environment of the workstation was able to guarantee the fast calculation of the algorithm model. The algorithm model was built in the TensorFlow framework using Python 3.5.

After the algorithm model was built, the preprocessed data were imported into the model for training. First, according to the ID and relative position of the lanechanging vehicle, vehicles that may affect the lane-changing vehicle within a certain range (set to $100 \mathrm{~m}$ ) were identified. According to the payoff function of the game theory model, the payoff value of each vehicle was calculated. The model outputs the possibility of vehicle lane changing and adds it as a data dimension to the vehicle driving-state data. Then, the data with the possibility of lane changing and the segmented lane-changing behavior samples were input to the deeplearning algorithm model. Among the samples, 70\% were used for training, and 30\% were used for verification. After many tests, the network model parameters were adjusted to the optimal state. The LSTM structure contains a hidden 
TABLe 2: Descriptive statistics of selected dataset.

\begin{tabular}{|c|c|c|c|c|}
\hline Variable & Mean & Std. dev. & Min & $\operatorname{Max}$ \\
\hline Speed $(\mathrm{m} / \mathrm{s})$ & 9.25 & 4.03 & 0 & 29.05 \\
\hline Acceleration $\left(\mathrm{m} / \mathrm{s}^{2}\right)$ & 0.05 & 1.27 & -3.01 & 2.95 \\
\hline Space headway $(\mathrm{m})$ & 19.59 & 9.51 & 0 & 52.79 \\
\hline Time headway (s) & 2.86 & 2.09 & 0 & 29.83 \\
\hline
\end{tabular}

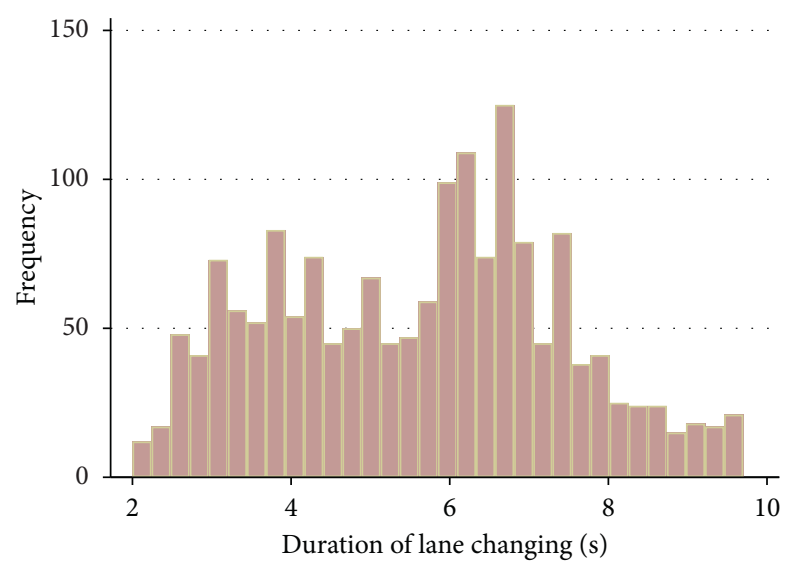

FIgURE 5: Duration of lane-changing process.

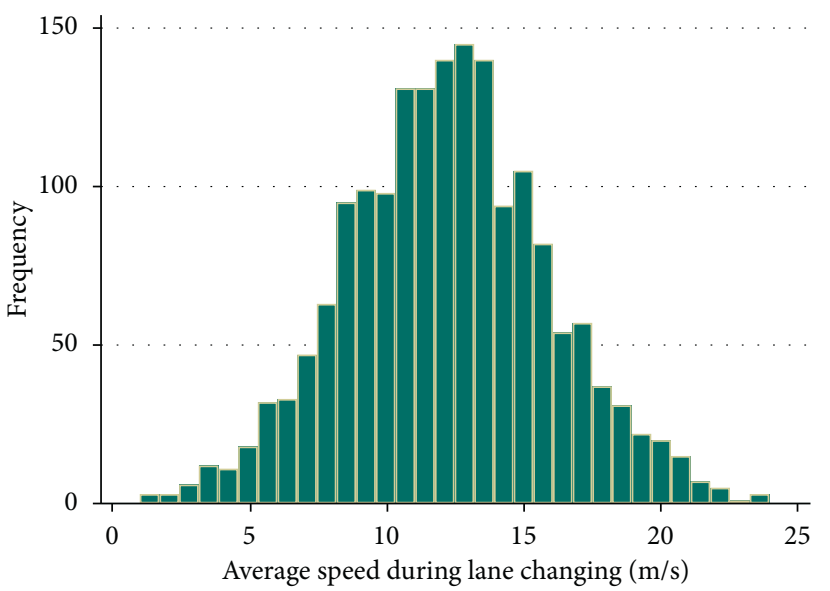

(a)

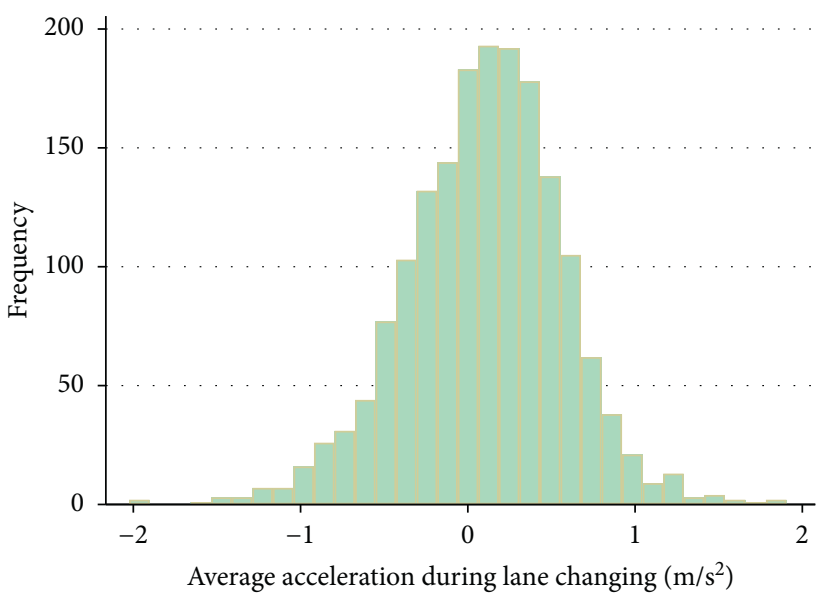

(b)

Figure 6: Average speed (a) and acceleration (b) during lane changing.

layer with 100 units. The input data are continuous drivingstate data combined with the lane-changing probability output obtained from the game theory model, and after extracting features, $100 \times 200$ data matrices are output for use as the input of the CNN module. Finally, the CNN module outputs the prediction results in real time, including lane keeping, left lane changing, and right lane changing. The learning rate was set to 0.001 , the time step was set to 3 , and the batch size was set to 8 . In the convolution layer of the $\mathrm{CNN}$, the convolution kernel was a $3 \times 3$ matrix, and the convolution step was 1 . The input data include lateral velocity, lateral acceleration, longitudinal velocity, longitudinal acceleration, time headway, headway, steering angle, and output of the game model.
During the training process, the optimizer in the TensorFlow framework is used to optimize the model. After 40000 model iterations, it tends to be optimal. The prediction result of the model is shown in Figure 7, and the prediction accuracy of the lane-changing behavior is maintained at approximately $94 \%$. Additionally, the change in the loss function in the training process is shown in Figure 8 . The lower the loss function, the closer the predicted status of lane changing is to the real status.

To verify the impact of the game theory model on the deep-learning model, we added a set of comparative tests and deleted the output results of the game theory model; that is, we directly used the vehicle's driving-state data to predict whether the vehicle will perform lane-changing operations. 


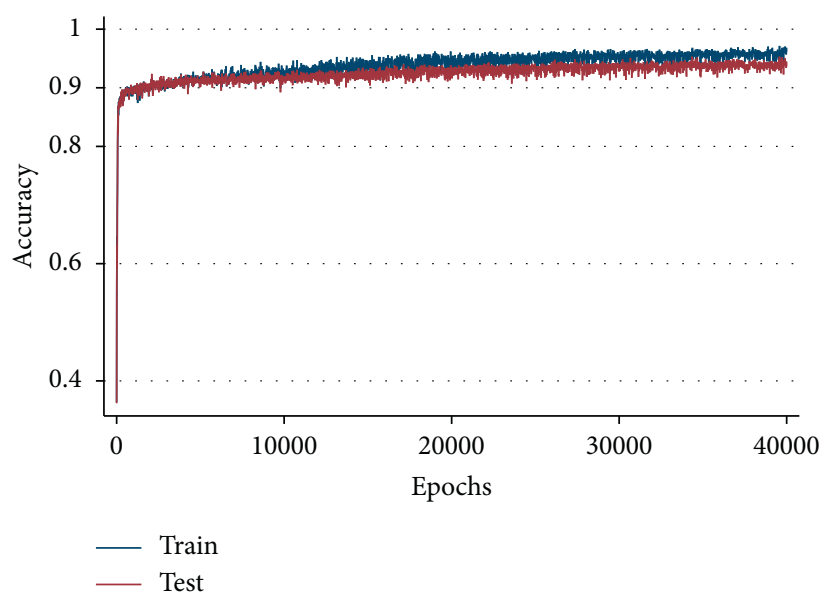

Figure 7: Prediction accuracy of LSTM-CNN model.

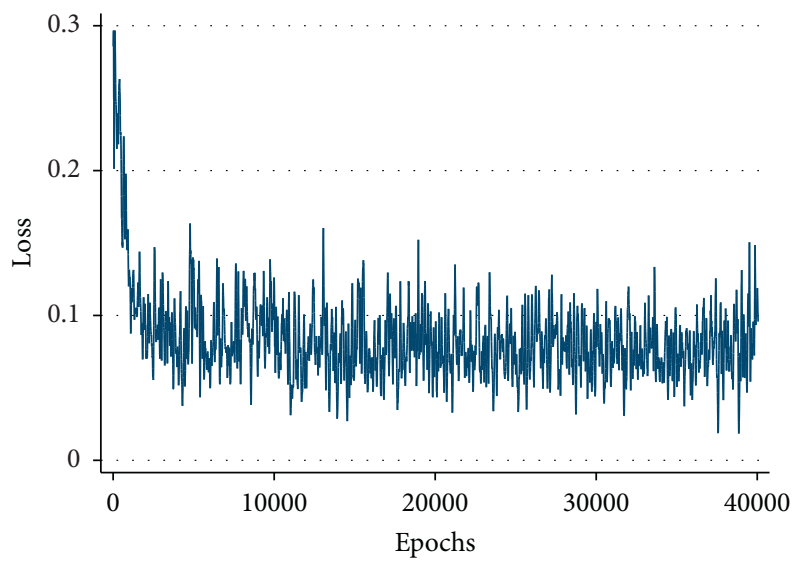

FIGURE 8: Loss function values of LSTM-CNN model.

The results of this experiment are shown in Figure 9. It can be seen that the accuracy of the deep-learning model in predicting vehicle lane changing decreases to approximately $86 \%$ after excluding the game theory results. Thus, it is proved that the game theory model in this study can effectively improve the prediction accuracy of lane changing.

In addition, to illustrate the prediction effectiveness of the model proposed in this paper, we use other commonly used algorithmic models to train and predict the same dataset for comparison. The prediction effects are summarized in Table 3. By comparing with a radial basis function neural network (RBFNN), BP neural network (BPNN), CNN, CNN-LSTM, and LSTM network, it can be found that the accuracy of the LSTM-CNN network used in this study is better than those of other algorithm models.

For the evaluation of lane-changing behavior prediction, the time taken by the model to obtain the correct prediction result is also an important parameter to evaluate its effectiveness. With every $0.1 \mathrm{~s}$ reduction achieved by the algorithm, the system can warn the surrounding vehicles $0.1 \mathrm{~s}$ in advance, which can reduce the occurrence of traffic accidents caused by lane-changing behavior to a certain extent. The time taken by our model to obtain the

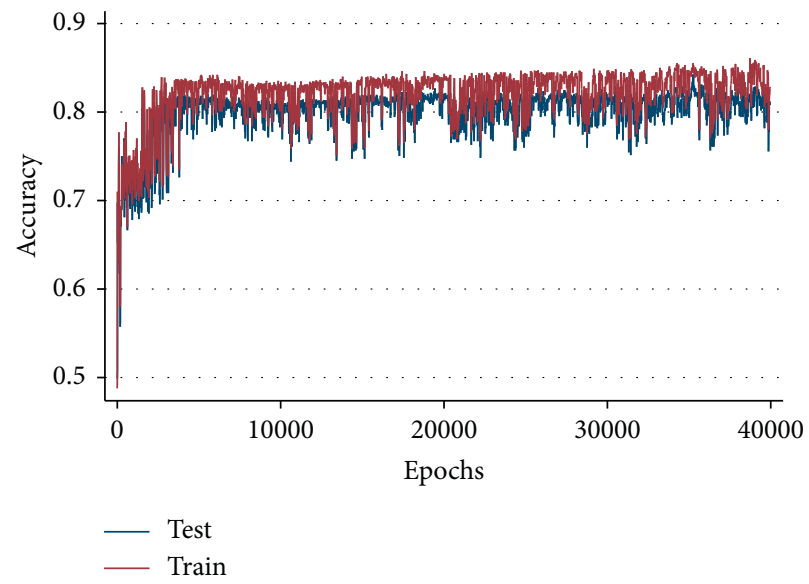

FIgURE 9: Prediction accuracy of the LSTM-CNN model without game theory results.

correct prediction results for lane-changing behavior is shown in Figure 10. It can be seen that the time spent is mostly between 0.2 and 0.4 seconds, indicating that the model can predict the lane-changing behavior of vehicles quickly. 
TABle 3: Prediction accuracy of different algorithm models.

\begin{tabular}{lcccc}
\hline $\begin{array}{l}\text { Algorithm } \\
\text { model }\end{array}$ & $\begin{array}{c}\text { Number of hidden } \\
\text { layers }\end{array}$ & $\begin{array}{c}\text { Number of } \\
\text { parameters }\end{array}$ & $\begin{array}{c}\text { Prediction accuracy with game } \\
\text { theory (\%) }\end{array}$ & $\begin{array}{c}\text { Prediction accuracy without game } \\
\text { theory }(\%)\end{array}$ \\
\hline RBFNN & 1 & 350 & 86.31 & 69.55 \\
BPNN & 2 & 63 & 84.89 & 75.36 \\
CNN & 2 & 48003 & 89.35 & 82.40 \\
LSTM & 1 & 167200 & 92.17 & 83.45 \\
CNN-LSTM & $2+1$ & 90048 & 92.65 & 82.12 \\
LSTM-CNN & $1+1$ & 167803 & 94.56 & 86.17 \\
\hline
\end{tabular}

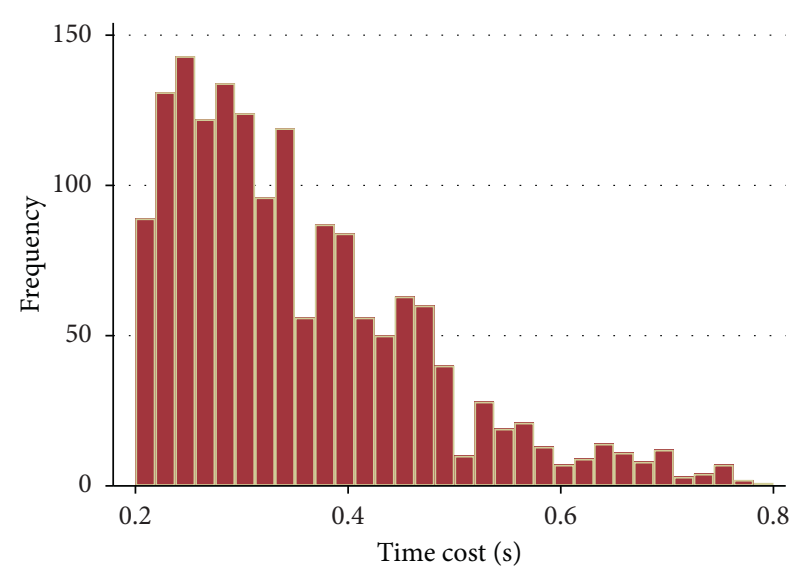

FIgURE 10: Time cost to obtain the correct prediction result.

\section{Conclusions and Future Work}

In this study, we proposed a prediction model of vehicle lane-changing behavior based on game theory and deep learning. The proposed model uses the concept of game theory to analyze the interaction between the target vehicle and surrounding vehicles and imports the driving status of multiple vehicles into the mathematical model to calculate the vehicle's intention to change lanes. Then, a deeplearning algorithm is used to integrate the vehicle driving state and the intention and accurately predict the vehicle lane-changing behavior. This study used NGSIM data to test the proposed model by extracting samples of lane changing from the original data and selecting some as training samples to optimize the parameters of the deeplearning network. The proposed model achieved a prediction accuracy of approximately $94.56 \%$, and most of the correct prediction results were obtained between 0.2 and $0.4 \mathrm{~s}$. In addition, to enhance the rationality of the experiment, we used the same dataset to compare the prediction accuracy of our model with those of the LSTM, CNN-LSTM, CNN, RBFNN, and BPNN algorithms. We also conducted a prediction experiment without the results of the game theory model. The experimental results demonstrate that our model has better performance and prove its efficiency and reliability.

In the future, to reduce the possibility of traffic accidents caused by driving behavior, we will consider more detailed vehicle behaviors, conduct trajectory prediction, and determine whether the vehicle is in a dangerous environment.

\section{Data Availability}

The data used to support the research work of this study are included within the article.

\section{Conflicts of Interest}

The authors declare that there are no conflicts of interest regarding the publication of this paper.

\section{Acknowledgments}

This work received funding support from the National Key Research and Development Program of China (no. 2018YFB1600604), the S\&T Program of Hebei (no. 20470801D), the Key Research and Development Program of Shaanxi Province (nos. 2018ZDCXLGY-04-02 and 2018ZDCXLGY-05-01), and the Fundamental Research Funds for the Central Universities (no. 300102249503).

\section{References}

[1] C. Saiprasert and W. Pattara-Atikom, "Smartphone enabled dangerous driving report system," in Proceedings of the 2013 46th Hawaii International Conference on System Sciences (HICSS), pp. 1231-1237, IEEE, Wailea, HI, USA, January 2013.

[2] S. E. Lee, E. C. Olsen, and W. W. Wierwille, "A comprehensive examination of naturalistic lane-changes," National Highway Traffic Safety Administration, Washington, DC, USA, DOT HS 809702, 2004.

[3] R. Dang, F. Zhang, J. Wang, S. Yi, and K. Li, "Analysis of Chinese driver's lane change characteristic based on real vehicle tests in highway," in Proceedings of the 16th International IEEE Conference on Intelligent Transportation Systems (ITSC 2013), pp. 1917-1922, IEEE, The Hague, Netherlands, October 2013.

[4] C. Macadam, Z. Bareket, P. Fancher, and R. Ervin, "Using neural networks to identify driving style and headway control behavior of drivers," Vehicle System Dynamics, vol. 29, no. sup1, pp. 143-160, 1998.

[5] Z. Zheng, "Recent developments and research needs in modeling lane changing," Transportation Research Part B: Methodological, vol. 60, pp. 16-32, 2014.

[6] P. G. Gipps, "A model for the structure of lane-changing decisions," Transportation Research Part B: Methodological, vol. 20, no. 5, pp. 403-414, 1986.

[7] Q. Yang and H. N. Koutsopoulos, "A microscopic traffic simulator for evaluation of dynamic traffic management systems," Transportation Research Part C: Emerging Technologies, vol. 4, no. 3, pp. 113-129, 1996. 
[8] J. Li-Sheng, F. Wen-Ping, Z. Ying-Nan, Y. Shuang-Bin, and H. Hai-Jing, "Research on safety lane change model of driver assistant system on highway," in Proceedings of the IEEE Intelligent Vehicles Symposium, pp. 1051-1056, IEEE, Xi'an, China, June 2009.

[9] V. Butakov and P. Ioannou, "Personalized driver/vehicle lane change models for ADAS," IEEE Transactions on Vehicular Technology, vol. 64, no. 10, pp. 4422-4431, 2015.

[10] Q. H. Do, H. Tehrani, S. Mita, M. Egawa, K. Muto, and K. Yoneda, "Human drivers based active-passive model for automated lane change," IEEE Intelligent Transportation Systems Magazine, vol. 9, no. 1, pp. 42-56, 2017.

[11] M. Keyvan-Ekbatani, V. L. Knoop, and W. Daamen, "Categorization of the lane change decision process on freeways," Transportation Research Part C: Emerging Technologies, vol. 69, pp. 515-526, 2016.

[12] M. Kamrani, A. R. Srinivasan, S. Chakraborty, and A. J. Khattak, "Applying markov decision process to understand driving decisions using basic safety messages data," Transportation Research Part C: Emerging Technologies, vol. 115, Article ID 102642, 2020.

[13] H. Jin, C. Duan, Y. Liu, and P. Lu, "Gauss mixture hidden markov model to characterise and model discretionary lanechange behaviours for autonomous vehicles," IET Intelligent Transport Systems, vol. 14, no. 5, pp. 401-411, 2020.

[14] G. B. Singh and H. Haiping Song, "Using hidden markov models in vehicular crash detection," IEEE Transactions on Vehicular Technology, vol. 58, no. 3, pp. 1119-1128, 2009.

[15] W. Wang, J. Xi, and D. Zhao, "Learning and inferring a driver's braking action in car-following scenarios," IEEE Transactions on Vehicular Technology, vol. 67, no. 5, pp. 3887-3899, 2018.

[16] C. Song, X. Yan, N. Stephen, and A. A. Khan, "Hidden markov model and driver path preference for floating car trajectory map matching," IET Intelligent Transport Systems, vol. 12, no. 10, pp. 1433-1441, 2018.

[17] Y. Qi and S. Ishak, "A hidden markov model for short term prediction of traffic conditions on freeways," Transportation Research Part C: Emerging Technologies, vol. 43, pp. 95-111, 2014.

[18] Y. Ali, Z. Zheng, M. M. Haque, and M. Wang, "A game theory-based approach for modelling mandatory lanechanging behaviour in a connected environment," Transportation Research Part C: Emerging Technologies, vol. 106, pp. 220-242, 2019.

[19] A. Talebpour, H. S. Mahmassani, and S. H. Hamdar, "Modeling lane-changing behavior in a connected environment: a game theory approach," Transportation Research Part C: Emerging Technologies, vol. 59, pp. 216-232, 2015.

[20] M. Wang, S. P. Hoogendoorn, W. Daamen, B. Van Arem, and R. Happee, "Game theoretic approach for predictive lanechanging and car-following control," Transportation Research Part C: Emerging Technologies, vol. 58, pp. 73-92, 2015.

[21] H. Yu, H. E. Tseng, and R. Langari, "A human-like game theory-based controller for automatic lane changing," Transportation Research Part C: Emerging Technologies, vol. 88, pp. 140-158, 2018.

[22] X. Di, X. Chen, and E. Talley, "Liability design for autonomous vehicles and human-driven vehicles: a hierarchical gametheoretic approach," Transportation Research Part C: Emerging Technologies, vol. 118, Article ID 102710, 2020.

[23] J. Yu, Z. Chen, Y. Zhu, Y. Chen, L. Kong, and M. Li, "Finegrained abnormal driving behaviors detection and identification with smartphones," IEEE Transactions on Mobile Computing, vol. 16, no. 8, pp. 2198-2212, 2016.

[24] Z. Zhao, W. Chen, X. Wu, P. C. Y. Chen, and J. Liu, "LSTM network: a deep learning approach for short-term traffic forecast," IET Intelligent Transport Systems, vol. 11, no. 2, pp. 68-75, 2017.

[25] J. Cheng, K. Suzuki, and M. Fujita, "Predicting driver's lanechanging decisions using a neural network model," Simulation Modelling Practice \& Theory International Journal of the Federation of European Simulation Societies, vol. 42, pp. 7383, 2014.

[26] J. Peng, Y. Guo, R. Fu, W. Yuan, and C. Wang, "Multi-parameter prediction of drivers' lane-changing behaviour with neural network model," Applied Ergonomics, vol. 50, pp. 207-217, 2015.

[27] K. Li, X. Wang, Y. Xu, and J. Wang, "Lane changing intention recognition based on speech recognition models," Transportation Research Part C: Emerging Technologies, vol. 69, pp. 497-514, 2016.

[28] F. Fabiani and S. Grammatico, "Multi-vehicle automated driving as a generalized mixed-integer potential game," IEEE Transactions on Intelligent Transportation Systems, vol. 21, no. 3, pp. 1064-1073, 2019.

[29] J. Nash, "Non-cooperative games," The Annals of Mathematics, vol. 54, no. 2, pp. 286-295, 1951.

[30] F. Qiao, R. Rahman, Q. Li, and L. Yu, "Safe and environmentfriendly forward collision warning messages in the advance warning area of a construction zone," International Journal of Intelligent Transportation Systems Research, vol. 15, pp. 166179, 2016. 